\title{
Communication Energy Optimization of Electric Vehicle Platoon on Curved Road
}

Wei Gao ( $\square$ gaoweibupt@bupt.edu.cn )

Beijing University of Posts and Telecommunications https://orcid.org/0000-0002-4860-3519

\section{Celimuge Wu}

The University of Electro-Communications

\section{Research Article}

Keywords: platoon, electric vehicle, communication energy optimization, auxiliary energy systems

Posted Date: September 3rd, 2021

DOI: https://doi.org/10.21203/rs.3.rs-853175/v1

License: (c) (1) This work is licensed under a Creative Commons Attribution 4.0 International License. Read Full License 


\title{
Communication Energy Optimization of Electric Vehicle Platoon on Curved Road
}

\author{
Wei $\mathrm{Gao}^{1 *}$ and Celimuge $\mathrm{Wu}^{2}$
}

\begin{abstract}
The cruising range of an electric vehicle is limited by its battery. Reducing the energy consumption of MES (main energy systems) or AES (auxiliary energy systems) of the vehicle battery is an effective means to increase the electric vehicle cruising range. Platoon driving can greatly reduce the wind resistance of the vehicle and then reduce the energy consumption of MES for electric vehicles. This paper proposes an adaptive communication energy optimization scheme based on road curvature radius to save the energy of AES for the electric vehicle platoon on curved roads. In this paper, the inter-vehicle distance error based on the car-like model in a two-dimensional space is established. Then, the inter-vehicle distance error is used to design a control law $K$ to accomplish successful platooning. Next, three platooning control schemes based on different information flow topologies are discussed. Finally, the consensus of three platooning control schemes and the energy consumption of electric vehicle communication systems are analyzed by MATLAB's Simulink. Simulation results show that the communication energy optimization scheme reduces the power consumption of AES as long as the platoon driving on curved roads.
\end{abstract}

Keywords: platoon; electric vehicle; communication energy optimization; auxiliary energy systems
"Correspondence: gaoweibupt@bupt.edu.cn

${ }^{1}$ Beijing University of Posts and Telecommunications Beijing, the State Key Laboratory of Networking and Switching Technology, Beijing, China

Full list of author information is available at the end of the article

\section{Introduction}

Since electric vehicles (EVs) are considered as zero pollution for gaseous pollutants, it is one of the most important reasons for the development of EVs. However, compared to fuel vehicles, electric vehicles have short cruising distances. Electric vehicle platoon is a good way to improve the cruising range of vehicles. Platoon can greatly reduce wind resistance. But, the frequent communication among platoon members increases the power consumption of auxiliary energy systems for vehicle batteries.

Some auxiliary energy systems (AES), including necessary lighting, heating, and communication are directly related to the platooning cruise range [1]. Other systems are in the main energy systems (MES) such as power steering, brake booster, air conditioner, satellite navigation, etc. All of them use electrical energy from batteries thus reduce vehicle cruising range. Among them, wireless communication of AES is usually considered as an important element of platooning.

Vehicle platooning is a group of vehicles in a close manner through wireless communication to achieve safe and high-speed driving [2] [3]. Control and computing technologies are integrated together to achieve stability, consensus, scalability, reliability, efficiency, and safety of the platooning system by exchanging vehicle information (such as speed, heading and intentions, etc.) over wireless networks to maintain relatively small inter-vehicle distance [4-7]. Frequent information interaction consumes a lot of battery power and reduces the cruising range of electric vehicle platoon.

Cruising range is one of the main obstacles of electric vehicles. In order to overcome it without increasing the battery size and price of the vehicle, one solution is to conduct energy optimization. Most existing electric vehicle energy optimization methods are conducted for MES, AES or a collaboration between the two. Thibault et al. propose model-based strategies to predict and optimize the energy consumption of a trip [8]. Zhang et al. combine terraininformation and preceding-vehicle information in energy management [9]. Xu et al. present two braking regenerative energy optimization controllers for in-wheel motor electric vehicles to achieve the braking requirement as well as effectively promote regenerative efficiency [10]. The above studies save driving energy of MES with vehicle movement 
prediction to prolong the driving distance for an electric vehicle. In the search for better efficiency, AES for EV is designed. A predictive decision support system is designed to optimally distribute energy flow between the instantaneous power demand requested by the driver for the powertrain engine and the AES such as the heating system [11]. An ultracapacitor-based AES is designed to greatly reduce the energy consumption of AES [1]. In addition to considering MES and AES separately, the joint optimization of these two systems can also increase vehicle travel distance. Jin et al. study on the problem of allocating energy from renewable sources to EV in a cost-efficient manner [12].

MES and AES are two main systems that determine the battery consumption level. Reducing the power consumption of subsystems in any system can increase the cruising range of vehicles. Under the premise of ensuring stable platoons, in order to reduce the battery consumption of electric vehicles, we propose a method to reduce the communication energy consumption in the AES. We observe that when platoons are driven on curved roads, the inter-vehicle distance and inter-vehicle communication distance are not equal. Regardless of the manner in which the platoon is formed, it is necessary to frequently exchange vehicle dynamics information between the vehicle and its preceding vehicles. Therefore, an adaptive radius of curvature communication energy allocation scheme is proposed to save the energy of AES on curved road.

The main contributions of this paper are as follows:

- Modelling of inter-vehicle distance error of platoon on curved road is constructed, and a constant control law $K$ is used to control the error. Simulation results show that an appropriate constant control law can maintain the platooning consensus.

- Typical platooning control schemes, namely, the decentralized control under PF (predecessor-following), decentralized control under PLF (predecessor-leader following), and centralized control by the leader, are discussed.

- When the platooning reaches a consensus, the proposed energy optimization scheme is superior to the minimum transmitted power scheme on straight road and the maximum radius of curvature scheme on curved road.

The rest of this paper is organized as follows. Communication energy optimization of electric vehicle platoon is proposed in section I. Methods include platoon under two-dimensional space, platooning control schemes, and curvature-driven communication mechanism are introduced in section II. Results are discussed in section III. Concluding remarks and future work are presented in section IV.

\section{Methods}

The car-like model is the basis for studying the platoon in a real environment [13]. The car-like model in the two- dimensional space quantifies the deviation of the vehicle from the road in a given two-dimensional space. In longitudinal control, the car-like model is used as a placement platform for the on-board laser and radar sensors to establish a mathematical model of the inter-vehicle distance. The placed sensor is used to detect the inter-vehicle distance, and then transmit the message to the platoon controller to maintain the constant time headway or constant distance strategy of the platoon, thereby ensuring the longitudinal safety of the platoon. For example, Godbole and Lygeros propose the longitudinal control laws to maintain safe spacing, track an optimal velocity and perform various maneuvers (forming, breaking up platoons, and changing lanes) [14]. In the lateral control, the car-like model is usually used to establish the relationship between the steering angle and the road offset, and to calculate the optimal steering angle when the vehicle is turning. Then the vehicle maintains the distance between the nearest point and the curved road to ensure the lateral safety of the platoon. When driving on a curved road, the platoon includes both longitudinal control and lateral control. For instance, Wei et al. realize the longitudinal and lateral vehicle following only by radar and V2V, independent of high-accuracy positioning system and road marking [15]. Bayuwindra et al. overcome cut corners by the look-ahead is extended to a point perpendicular to the direction of the preceding vehicle and then present a novel look-ahead concept for combined longitudinal and lateral vehicle following control for a car-like platoon [16]. Finally, establishing the lateral deviation under the car-like model is a necessary condition for establishing the longitudinal inter-vehicle distance error.

\section{1 platoon under two-dimensional space}

The curved road with varying curvature is established under the two-dimensional space for vehicle platooning. This method is close to the actual road design and easy to implement in the program. The movement of the platoon in the two-dimensional space causes a change in the inter-vehicle distance. At this time, the platoon stability varies with the error in the inter-vehicle distance for platooning control. Typically, inter-vehicle distance errors in the platoon are related to changes in road curvature and control law.

The curved road having different curvatures are designed, and curves of different curvatures are connected into curved roads by adjusting curvature control points. Then, we build a car-like model in the two-dimensional space. Then, the inter-vehicle distance error is established under the curvature space, and a constant control law $K$ is added to design a stable platoon.

\subsubsection{Car-like model}

Car-like model is composed of a motorized wheeled axle at the rear of the chassis, and a pair of orientable front steering wheels. For an easier analytical representation of the 
Car-like model, we put this model into a two-dimensional space, as represented in Fig. 1. X and Y represent the horizontal and vertical axes of the Cartesian coordinate system, respectively. The simple kinematic model is described as follows:

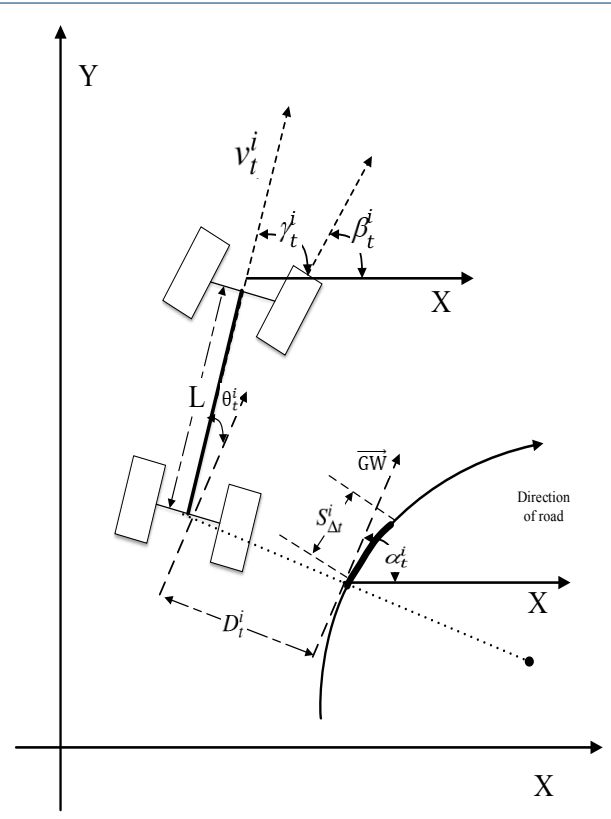

Figure 1 Car-like model.

$$
\begin{aligned}
& \dot{x}_{i}=v_{t}^{i} \cdot \cos \gamma_{t}^{i}, \\
& \dot{y}_{i}=v_{t}^{i} \cdot \sin \gamma_{t}^{i}, \\
& \dot{\gamma}_{t}^{i}=\frac{v_{t}^{i}}{L} \cdot \tan \left(\gamma_{t}^{i}-\beta_{t}^{i}\right),
\end{aligned}
$$

where $v_{t}^{i}$ is the velocity of vehicle $i$ at time $t . L$ is the wheelbase of the vehicle. $\gamma_{t}^{i}$ is the angle between orientation of vehicle $i$ at time $t$ and horizontal line with respect to world frame. $\beta_{t}^{i}$ is the steering angle of vehicle $i$ at time $t$ with respect to world frame. $\alpha_{t}^{i}$ is the angle of between orientation of the tangent to the trajectory at the same point in relation and horizontal line to the absolute reference. $S_{\Delta t}^{i}$ is the curved distance of vehicles $i$ from time $t$ to $t+\Delta t$. Given that Cartesian distance is not monotonous for very curved trajectories while quantifying the spacing between two consecutive vehicles, it is necessary to construct the model in curvilinear space, that is, with respect to the curvilinear abscissa of the nearest point on the trajectory measured from the vehicle, as shown follows.

$\overrightarrow{G W}$ represents the projection of the Cartesian velocity of the vehicle into curvilinear space. The vehicle and the road are offset from the angle of $\theta_{t}^{i}=\gamma_{t}^{i}-\alpha_{t}^{i} . c_{i}$ stands for the respective local curvature of vehicle $i$, which corresponds to the reciprocal of the radius of curvature. $\dot{S}_{\Delta t}^{i}$ is the further projection by considering the lateral deviation $D_{t}^{i}$.

$$
\begin{aligned}
& \dot{S}_{\Delta t}^{i}=\frac{v_{t}^{i} \cos \theta_{t}^{i}}{1+D_{t}^{i} c_{i}}, \\
& \dot{D}_{t}^{i}=v_{t}^{i} \cdot \sin \theta_{t}^{i}, \\
& \dot{\theta}_{t}^{i}=v_{t}^{i}\left(\frac{\tan \left(\gamma_{t}^{i}-\beta_{t}^{i}\right)}{L}-\frac{c_{i} \cdot \cos \theta_{t}^{i}}{1+c_{i} D_{t}^{i}}\right) .
\end{aligned}
$$

Proof: See Appendix A.

\subsubsection{Modelling of inter-vehicle distance error}

A longitudinal platooning system can be considered as a combination of four important components: vehicle longitudinal dynamics, information exchange flow, decentralized or centralized controllers and inter-vehicle distance policies [20] [27] [28] [29]. In order to analyze longitudinal inter-vehicle distance error, the assumptions of the other components are as follows:

(1) Vehicle longitudinal dynamics include the engine, drive line, brake system, aerodynamics drag, etc. In this paper, we consider that every vehicle has the homogeneous double-integrator model [30].

(2) Decentralized or centralized controller is adopted in this paper. Decentralized control is that each vehicle controls its own vehicle status based on other vehicles' information. Centralized control is that all platoon members are controlled by leader.

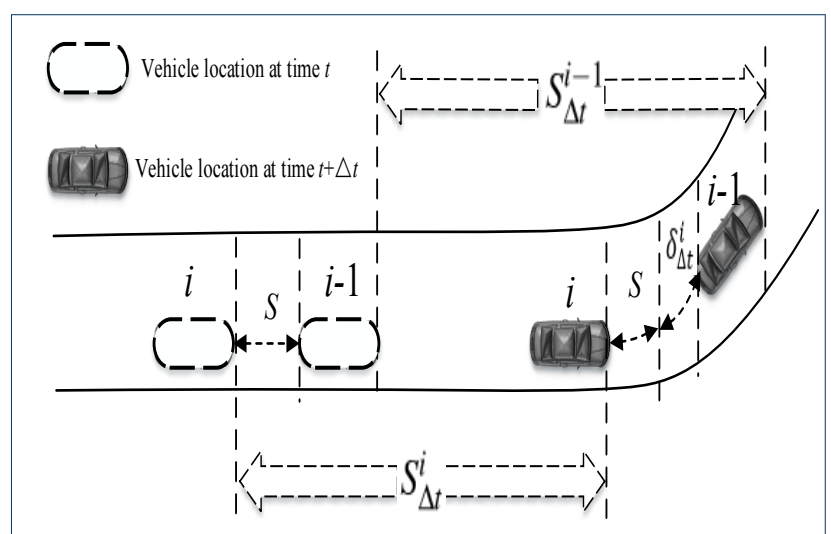

Figure 2 Distance error dynamic.

Considering $N$ homogeneous vehicles driving along the curved road, platoon member (vehicle $i$ ) is to follow its preceding vehicle driving at a desired inter-vehicle distance $S$. Two platoon members are schematically depicted in Fig. 2 
with $S$ being the distance between vehicle $i$ and its preceding vehicle $i-1$. The main objective of each vehicle is to follow its preceding vehicle at a desired distance $S$. But, inter-vehicle distance will be changed due to sensor delay, communication delay, weather and curved road, etc. causing a distance error $\delta_{\Delta t}^{i}$. Here $S_{\Delta t}^{i}$ and $S_{\Delta t}^{i-1}$ are the curved distance of vehicles $i$ and $i-1$ in time $\Delta t$, respectively. Let us define the curved distance error for the i-th vehicle as:

$$
\begin{aligned}
& \delta_{\Delta t}^{i}=S_{\Delta t}^{i-1}-S_{\Delta t}^{i}, \\
& \dot{\delta}_{\Delta t}^{i}=\dot{S}_{\Delta t}^{i-1}-\dot{S}_{\Delta t}^{i} .
\end{aligned}
$$

When $\Delta t \rightarrow 0$, combining equation (3b) with equation (2c), we can get the following equation.

$$
\dot{\delta}_{\Delta t}^{i}=\frac{v_{t}^{i-1} \cos \theta_{t}^{i-1}}{1+D_{t}^{i-1} c_{i-1}}-\frac{v_{t}^{i} \cos \theta_{t}^{i}}{1+D_{t}^{i} c_{i}}
$$

let $\dot{\delta}_{\Delta t}^{i}=K \cdot \delta_{\Delta t}^{i}$ and $K>0$. The expression of vehicle speed is as follows:

$$
v_{t}^{i}=\frac{1+D_{t}^{i} c_{i}}{\cos \theta_{t}^{i}}\left(\frac{v_{t}^{i-1} \cos \theta_{t}^{i-1}}{1+D_{t}^{i-1} c_{i-1}}-K \delta_{\Delta t}^{i}\right),
$$

where the vehicle speed $v_{t}^{i-1}$ and cured distance error $\delta_{\Delta t}^{i}$ are used as the control input of vehicle $i$.

In this section, the inter-vehicle distance error is converged by constant control law $K$ to keep the desired intervehicle distance. In the next section, the cooperation between multiple vehicles based on different information flow topologies is discussed.

\section{2 platooning control schemes}

For multi-vehicle platooning, different controller placement positions will lead to different information flow topologies. In other words, different information flow topologies have different suitable platoon controller placement positions. For example, the PF (predecessor-following) topology is a topology that does not obtain information about the dynamics of the leader. It is suitable for placing the controller on each vehicle of the platoon members, and the vehicle itself will calculate the desired inter-vehicle distance at each moment. Ghasemi et al. propose a hierarchical platoon controller design framework, where the second layer is composed of a decentralized bidirectional control controller [17]. Due to the lack of dynamics about the leader, the platoon is likely to lose stability as the number of vehicles increases [18]. In the PLF (predecessorleader following) topology, each vehicle of the platoon can receive broadcast information from the leader, so the controller can be placed in each vehicle or only in the leader.
When the controller is only placed in the leader, the leader controls platoon members through the network, which is called a centralized control platoon. When the controller is placed in each vehicle, the leader only broadcasts its dynamics information to platoon members, which is a decentralized control platoon. In paper [19], a centralized and distributed control policy is proposed in which each vehicle's control decision depends solely on its relative kinematics with respect to the leader. In paper [20], for each topology, Chehardoli et al. propose a new neighbor-based adaptive control law to deal with adaptive control and identification of 1-D platoon of non-identical vehicles. Therefore, platoon control schemes under multi-vehicle mainly focus on the control and communication topology.

Typical platooning information flow topologies include predecessor-following (PF) topology, predecessor-leader following (PLF) topology, bidirectional (BD) topology, bidirectional-leader (BDL) topology, two-predecessors following (TPF) topology, and two-predecessor-leader following (TPLF) topology [31] [32]. These information flow topologies are classified from the mode of transmitting the information. The simplex mode includes PF, PLF, TPF, and TPLF, while the duplex mode includes BD and BDL. In simplex mode, TPF and TPLF extend from PF and PLF, respectively. Therefore, the most dominant topologies are PF and PLF. The information flow topology only shows the transmission path of the platooning information and must be combined with platooning control strategy to stabilize the platoon.

Platooning control strategies can be centralized or decentralized according to the position of the controller [33]. In the centralized control, vehicles get their control commands from central units. They are therefore not autonomous and communication is fundamental: any loss or delay in communication is critical. While in decentralized control, each vehicle receives data from other vehicles, and calculates its own control in a stand-alone manner, so that communication remains very important, but that its loss is not as critical as the centralized case [34]. In general, the topology should match the control strategies.

Combining platooning information flow topologies and platooning control strategies, three typical platooning control schemes are proposed in Fig. 3. The first vehicle of each platoon is called the leader, and the others are called platoon members. Platooning control scheme (a) is a combination of PF topology and decentralized control that platoon members themselves adjust the vehicular dynamics based on the received dynamic information of the preceding vehicle to maintain the desired distance between the vehicle and preceding vehicle. Platooning control scheme (b) is a combination of PLF topology and decentralized control that platoon members themselves adjust the vehicular dynamics based on the received dynamic information of the preceding vehicle and leader to maintain the desired distance between the vehicle and preceding vehicle. Scheme 


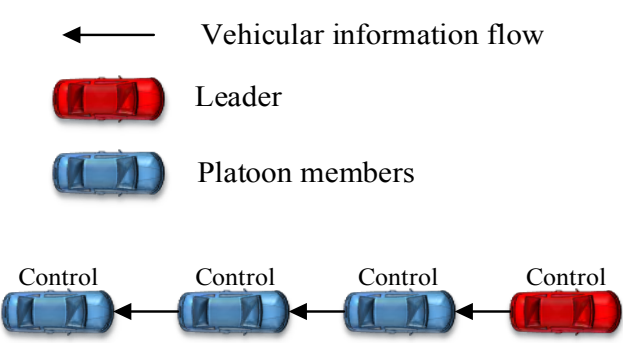

(a)

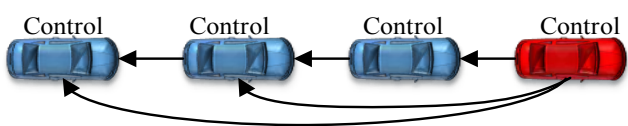

(b)

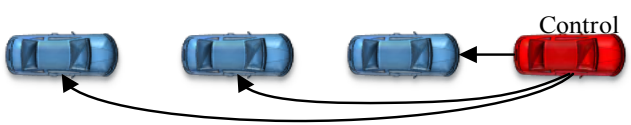

(c)

Figure 3 Typical platooning control schemes. (a) Decentralized control under PF. (b) Decentralized control under PLF. (c) Centralized control by leader.

(c) is centralized control approach by the leader that all platoon members adjust the speed according to the control commands message of the leader to maintain platooning stability.

There are two major spacing policies for the desired intervehicle distance $S$ : the constant time headway policy and constant distance policy [35]. For the constant time headway policy, the desired inter-vehicle distance varies with vehicle velocity. In the constant distance policy, the desired distance between two consecutive vehicles is independent of vehicle velocity. Here, we consider the constant time headway policy used for scheme (a), because the vehicles can only get the information of the predecessor. In order to ensure the safety of the vehicles, inter-vehicle distance needs to change with the speed of the predecessor. For scheme (b), we consider a constant distance, which means that the vehicles are controlled to move in a rigid platoon while following a leader because the vehicles can get the information of the predecessor and leader. Scheme (c) is similar to scheme (b) but lacks the preceding vehicle information.

When $S=T_{s} v_{t}^{i}$, the platooning control scheme (a) is structured as follows. This is constructed according to previous literature by Segata et al. [36].

$$
\ddot{S}_{i}^{d e s}(t+\Delta t)=-\frac{1}{T_{S}}\left(\dot{\xi}_{i}+\lambda \delta_{\Delta t}^{i}\right),
$$

where $\ddot{S}_{i}^{d e s}(t+\Delta t)$ is desired acceleration of vehicle $i$ at time $t+\Delta t, \lambda$ is a design parameter strictly greater than 0 (default set to 0.1 ). $\dot{\xi}_{i}=v_{t}^{i}-v_{t}^{i-1}$ is relative speed between vehicle $i$ and vehicle $i-1 . T_{s}$ is time headway. In order to ensure the safety of passengers, [35] shows that it must satisfies $T_{s} \geq 2 L$, where $L$ is actuation lag [37].

By combining Formula (5) and (6), then we get the following formula.

$$
\begin{aligned}
\ddot{S}_{i}^{d e s}(t+\Delta t)= & -\frac{v_{t}^{i-1}}{T_{S}}\left(\frac{\cos \theta_{t}^{i-1}\left(1+D_{t}^{i} c_{i}\right)}{\cos \theta_{t}^{i}\left(1+D_{t}^{i-1} c_{i-1}\right)}-1\right) \\
& -\frac{\delta_{\Delta t}^{i}}{T_{S}}\left(\lambda-\frac{k\left(1+D_{t}^{i} c_{i}\right)}{\cos \theta_{t}^{i}}\right) .
\end{aligned}
$$

When $S$ is the constant distance, the platooning control scheme (b) is structured as follows. This is derived based on a previous literature by Segata et al. [36],

$$
\begin{aligned}
\ddot{S}_{i}^{d e s}(t+\Delta t) & =a \ddot{S}_{i-1}(t)+b \ddot{S}_{0}(t) \\
& \left.+c \dot{\xi}_{i}+d\left(v_{t}^{i}-v_{t}^{0}\right)\right) \\
& +e \delta_{\Delta t}^{i} .
\end{aligned}
$$

By combining formula (5) and (8),

$$
\begin{aligned}
\ddot{S}_{i}^{d e s}(t+\Delta t) & =a \ddot{S}_{i-1}(t)+b \ddot{S}_{0}(t) \\
& +v_{t}^{i-1}\left(\frac{\cos \theta_{t}^{i-1}\left(1+D_{t}^{i} c_{i}\right)}{\cos \theta_{t}^{i}\left(1+D_{t}^{i-1} c_{i-1}\right)}(c+d)-c\right)-d v_{t}^{0} \\
& +\delta_{\Delta t}^{i}\left(e-(c+d) \frac{k\left(1+D_{t}^{i} c_{i}\right)}{\cos \theta_{t}^{i}}\right)
\end{aligned}
$$

where $v_{t}^{0}$ and $\ddot{S}_{0}(t)$ are the speed and acceleration of leader respectively. $a, b, c, d, e$ are parameters depicted as follows:

$$
\begin{aligned}
& a=1-W \\
& b=W \\
& c=-\left(2 \zeta-W\left(\zeta+\sqrt{\zeta^{2}-1}\right)\right) b_{w} \\
& d=-W\left(\zeta+\sqrt{\zeta^{2}-1}\right) b_{w} \\
& e=-b_{w}^{2}
\end{aligned}
$$

where $W$ is weighting factor between the acceleration of leader and preceding vehicle. $\zeta$ is drag coefficient which is set as $1 . b_{w}$ is receiving signal frequency. Since the distance between the leader and vehicle $i$ is different from that between the vehicle $i-1$ and vehicle $i$, the delay times of vehicle $i-1$ and leader transmitting information to the vehicle $i$ are different. 
For scheme (c), it does not receive the information of the preceding vehicle, and formula (8) is transformed into the following formula.

$$
\ddot{S}_{i}^{d e s}(t+\Delta t)=b \ddot{S}_{0}(t)+(c+d) v_{t}^{i}-d v_{t}^{0}+e \delta_{\Delta t}^{i} .
$$

The desired acceleration $\ddot{S}_{i}^{\text {des }}(t+\Delta t)$ of the vehicle $i$ can be used as the power input of the engine at time $t+\Delta t$ in the simulation program. The flow chart of the program is as follows:

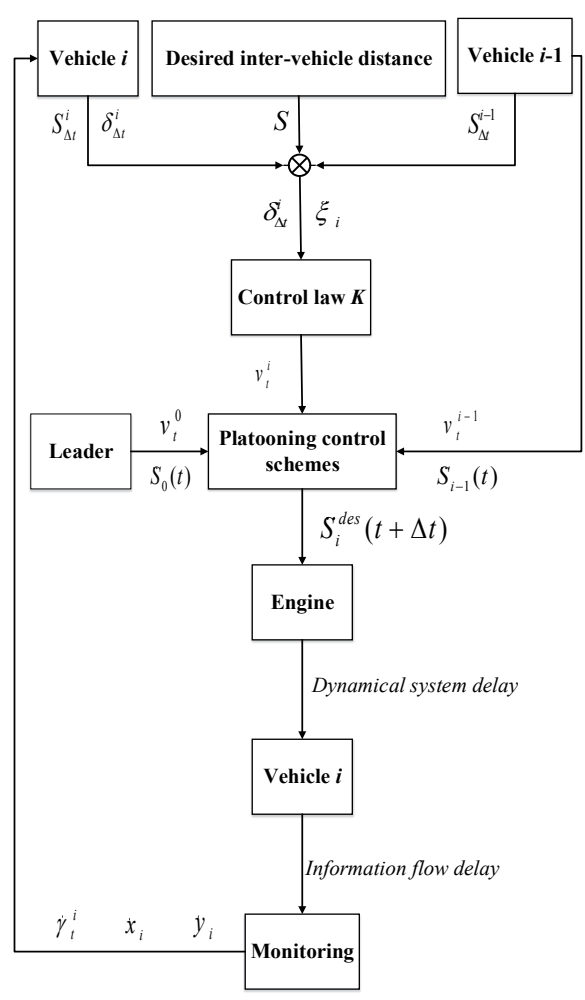

Figure 4 Flow chart of the platooning control schemes.

The flowchart is based on the travel time of the vehicle. This paper uses the speed and acceleration of vehicle $i-1$ and leader at time $t$ and the curved distance error $\delta_{\Delta t}^{i}$ within $\Delta t$ to calculate the desired acceleration $\ddot{S}_{i}^{\text {des }}(t+\Delta t)$ of the vehicle $i$ at time $t+\Delta t$.

The reason for curved distance error is the information flow delay and the dynamic system delay. The main reason for information flow delay is the difference in information flow topology. For example, PF topology can easily cause cascading delay. In the PLF topology, inter-channel interference and signal transmission failures cause repeated information transmission delays. The second cause of curved distance error is the dynamic system delay. That is caused by the vehicle's own mechanical transmission.

The main purpose of platooning control is to adjust the curved distance error $\delta_{\Delta t}^{i}$ according to the control law $k$ to make it tend to 0 . When the curve distance error $\delta_{\Delta t}^{i}$ is close to 0 , the control law $k$ has no effect on the speed, and a stable platoon is formed. In other words, the vehicle travels at a desired inter-vehicle distance. Then, when the platoon is driving on the curved road, the vehicle adjusts the transmission power of the OBU (On board Unit) according to the difference between the communication distance and the inter-vehicle curved distance. A curvature-driven communication mechanism is shown in the next section.

\section{3 curvature-driven communication mechanism}

Among the AES of electric vehicles, communication system is a continuous energy consumption part. The communication power control is a method to reduce the energy consumption of AES [21]. Communication energy includes both the power consumption for receive and send packets. Whether the power for the receiver or sender is reduced, the energy consumption of the AES communication system will be reduced, and the cruise range of the platoon will be increased. In the past, transmit power control (TPC) is usually used to improve the reliability and accuracy of communication. In [22], Zander investigates the control of co-channel and adjacent channels interference and achieves acceptable carrier-to-interference ratios by TPC in all active communication links in the system. Paper [23] describes a centralized power control scheme that computes transmitter powers so as to have a common carrier-to-interference ratio for all the receivers. In [24], Rosberg et al. use the transmitter power control techniques in protecting other users from excessive interference as well as making receivers more tolerant to this interference. In general, when the receiver's power is constant, the dynamic sensing power range requirement of the receiver is reduced and the adjacent channels are protected. At the same time, the transmitter's power control can reduce intercommunication interference and inter-channel interference. Therefore, besides improving the communication reliability and accuracy, communication power control can also be used to reduce AES energy consumption.

Communication modes among vehicles in the platoon include unicast, multicast, and broadcast. At this time, the signal transmission power conforms to the path loss model. The specific inter-vehicle communication distance model is as follows.

In the curve motion of the vehicle, the chord length $d$ corresponding to the arc length is $S+\delta_{\Delta t}^{i}$ as described in equation (12a). The proof of (12a) has been given in Appendix $\mathrm{B}$. Based on the basic $V 2 R$ path loss model given in [38] [39], we can get the following path loss equation (12b).

$$
d=\frac{2}{c_{i}} \cdot \sin \left(\frac{\left(S+\delta_{\Delta t}^{i}\right) \cdot c_{i}}{2}\right)
$$




$$
W_{i}=W_{i-1}+16.7 \log 10(d)+18.2 \log 10(f c),
$$

where $f c$ is in $G H z . W_{i}$ is the receive power from vehicle $i-1$ to vehicle $i$. When a stable platooning passes through the curved road with varying curvature, its intervehicle communication distance is different from that of the inter-vehicle curved distance. The difference between inter-vehicle curved distance and inter-vehicle communication distance is the starting point of Algorithm 1. According to this difference, the transmission power of the OBU is controlled to save the energy consumption of the communication system. Finally, a communication energy allocation algorithm for the adaptive radius of curvature is proposed.

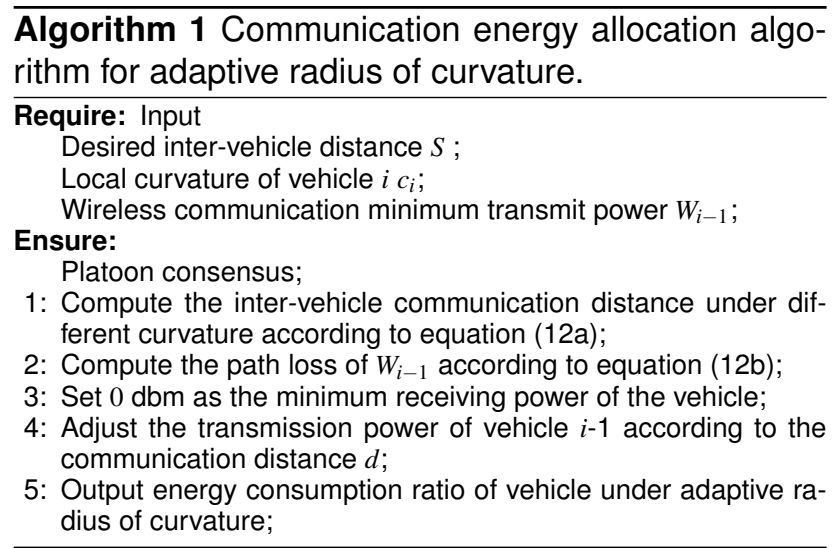

The general idea of Algorithm 1 is as follows, the preceding vehicle computes the inter-vehicle communication distance at every moment according to the change of road curvature. Then, the preceding vehicle adjusts the transmission power under the condition of meeting the minimum receiving power of the rear vehicle through the power control based on the radius of curvature. Before this algorithm, the curved distance error is computed according to the carlike model, and it is considered into the platooning control scheme. The platooning consensus is ensured by adjusting the control law $K$.

In Algorithm 1, the desired inter-vehicle distance is input into the algorithm as the initial communication distance. The local curvature is set according to the changes of the road. In the whole process of Algorithm 1, the platooning consensus must be ensured. In the first and second step of Algorithm 1, the minimum transmission power of vehicle $i-1$ relative to vehicle $i$ is set. The first step is to compute the inter-vehicle communication distance under the intervehicle curved distance, and the second step is to compute the minimum transmission power of vehicle $i-1$ according to the path loss model. The third step is to set the minimum receiving power of vehicle $i$ as $0 \mathrm{dBm}$. The fourth step is to adjust the transmission power of vehicle $i-1$ according to the change of inter-vehicle communication distance. In the fifth step, the ratio of vehicle energy consumption with adaptive curvature radius algorithm and without adaptive curvature scheme is output.

In summary, when the platooning is driving on the curved road, the communication distance and control distance of the inter-vehicle are different. For platooning, It is necessary to control the vehicles to maintain a desired intervehicle distance and achieve platooning consensus. Under platooning consensus, we adjust the transmission power of communication in real-time according to the inter-vehicle communication distance. In the case of ensuring the reliability of the communication link and platooning consensus, the energy consumption optimization of the communication system is the main contribution of this paper.

\section{RESULTS AND DISCUSSION}

Veins is an open source framework for vehicular network simulations [25]. PLEXE is an extension of the Veins simulator [26]. It features realistic vehicle dynamics and several cruise control models. In our simulation, we use MATLAB's Simulink tool to simulate platooning control schemes. Simulink is a block diagram environment for Model-Based Design. In the simulation process, we first establish the mathematical model of platooning control schemes and launch Simulink on the basis of the mathematical model. Simulink uses modular design to quickly and easily set dynamic parameters and build information flow topology for platoon.

Aiming at the communication energy optimization problem of electric vehicles, an adaptive road communication energy optimization method is proposed. In this section, we simulate the typical platooning control schemes. Then, according to the motion characteristics of vehicles, the consensus of the platoon is analyzed. Finally, an adaptive energy allocation scheme is established according to the intervehicle distance.

We consider a situation where a set of points with coordinates $p^{i}=\left[x^{i} y^{i}\right]^{T}$ with $i=1,2, \cdots, m$ ( $m$ is the number of points). These points will be henceforth control points of curved roads, so as to ascertain continuity in curvature and its derivative.

Table 1 Vehicle Parameters

\begin{tabular}{|c|c|c|c|}
\hline Typical platooning schemes & (a) & (b) & (c) \\
\hline Wheel base & $1 \mathrm{~m}$ & $1 \mathrm{~m}$ & $1 \mathrm{~m}$ \\
\hline Initial position (Leader) & {$\left[\begin{array}{lll}9 & 1\end{array}\right]^{T} m$} & {$\left[\begin{array}{lll}6 & 1\end{array}\right]^{T} m$} & {$\left[\begin{array}{ll}14 & 1\end{array}\right]^{T} m$} \\
\hline Initial orien & $0 \mathrm{rad}$ & $0 \mathrm{rad}$ & $0 \mathrm{rad}$ \\
\hline Initial positi & {$\left[\begin{array}{ll}5 & 1\end{array}\right]^{T} m$} & {$\left[\begin{array}{ll}3 & 1\end{array}\right]^{T} m$} & {$\left[\begin{array}{ll}11 & 1\end{array}\right]^{T} m$} \\
\hline Initial orientation (Follower 1) & $0 \mathrm{rad}$ & $0 \mathrm{rad}$ & $0 \mathrm{rad}$ \\
\hline Initial position (Follower 2) & {$\left[\begin{array}{lll}2 & 1\end{array}\right]^{T} m$} & {$\left[\begin{array}{lll}1 & 1\end{array}\right]^{T} m$} & {$\left[\begin{array}{ll}6 & 1\end{array}\right]^{T} m$} \\
\hline Initial orientation (Follower 2) & $0 \mathrm{rad}$ & $0 \mathrm{rad}$ & $0 \mathrm{rad}$ \\
\hline Initial position (Follower 3) & {$\left[\begin{array}{lll}0 & 1\end{array}\right]^{T} m$} & {$\left[\begin{array}{lll}0 & 1\end{array}\right]^{T} m$} & {$\left[\begin{array}{lll}0 & 1\end{array}\right]^{T} m$} \\
\hline Initial orien & $0 \mathrm{rad}$ & $0 \mathrm{rad}$ & $0 \mathrm{rad}$ \\
\hline Prol & 5 & 5 & $F$ \\
\hline Desired dis & $2 m$ & $2 m$ & $2 m$ \\
\hline
\end{tabular}




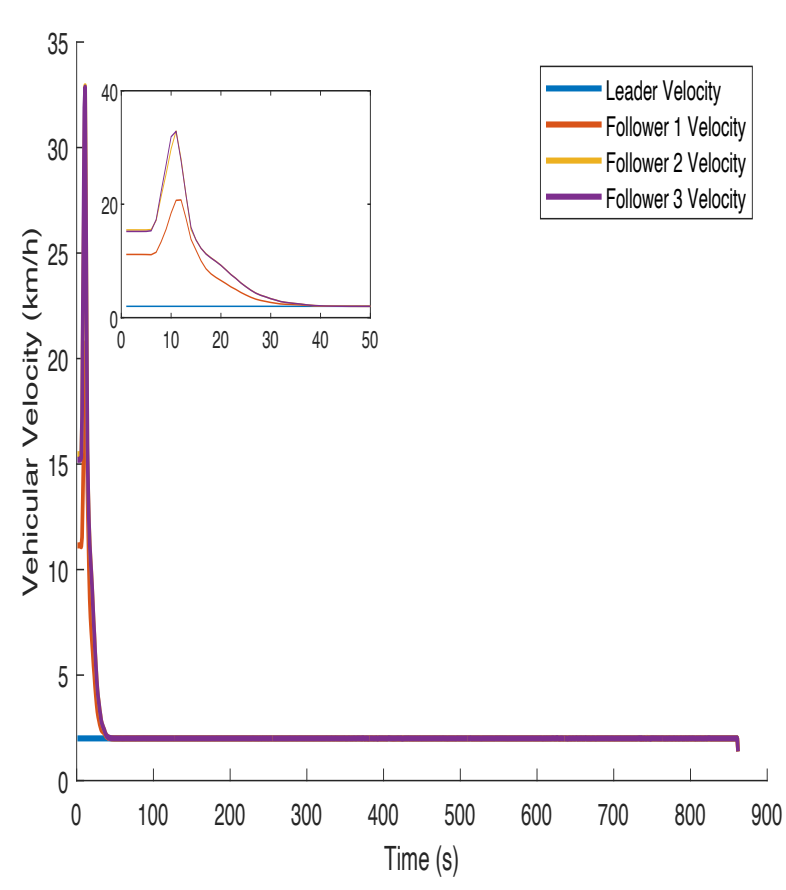

(a)

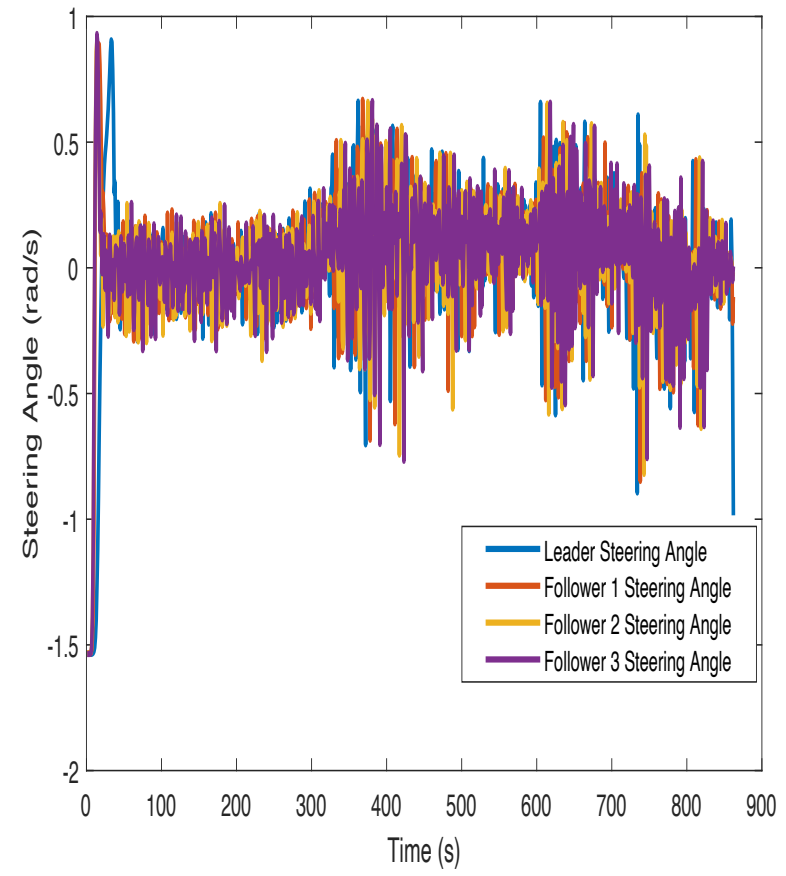

(c)

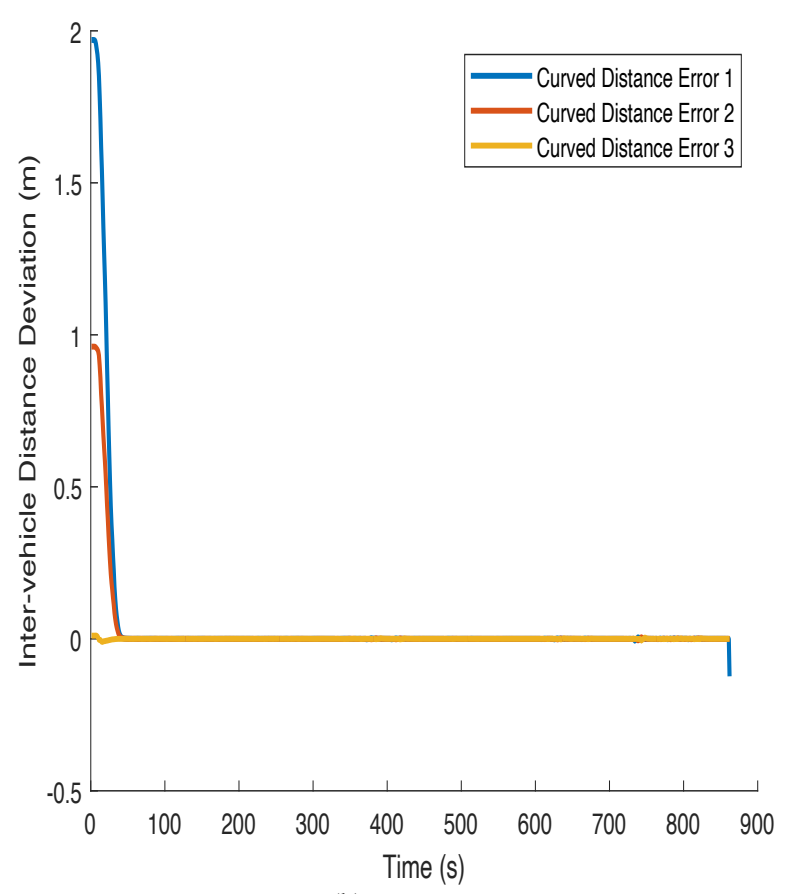

(b)

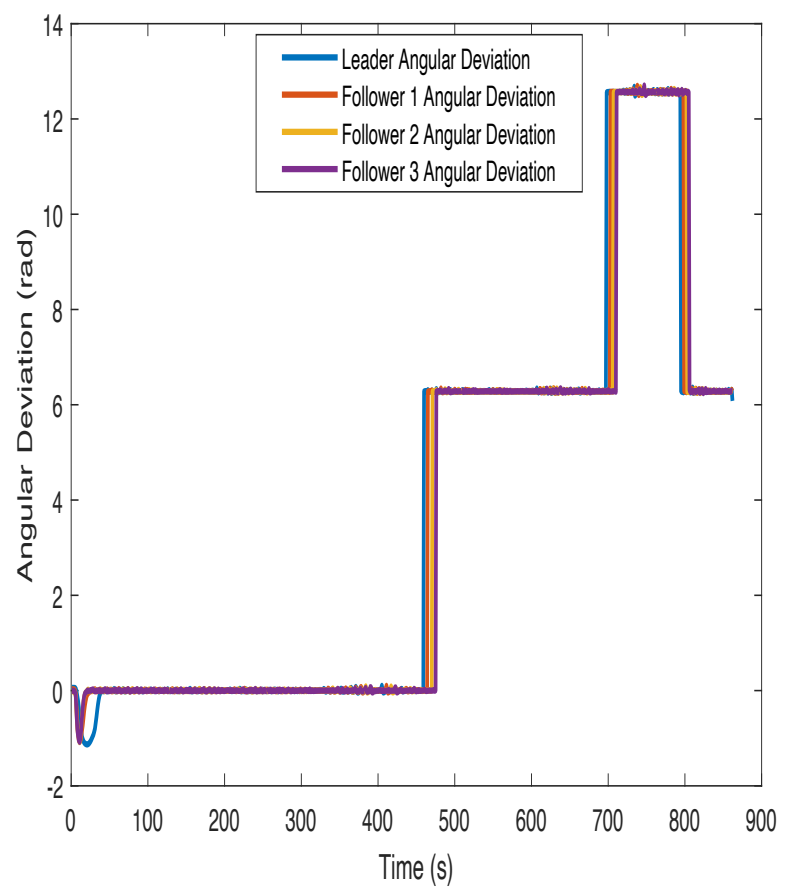

(d)

Figure 5 Decentralized control under PF: (a)Vehicular velocity; (b)Vehicular distance error; (c)Steering Angle; (d)Angular Deviation. 


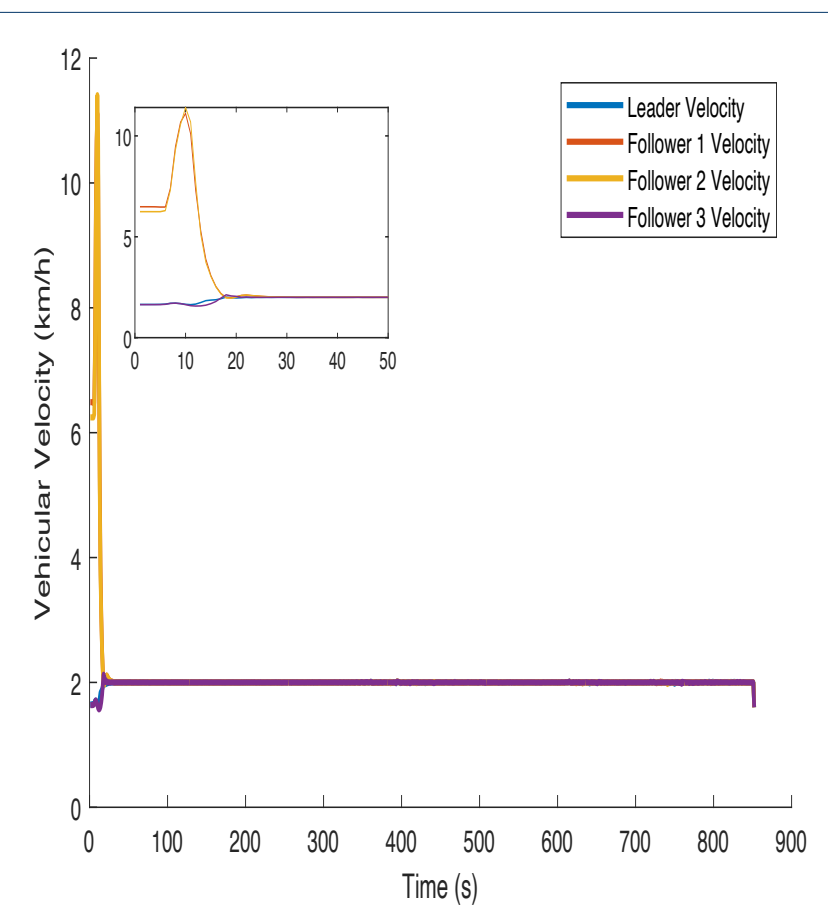

(a)

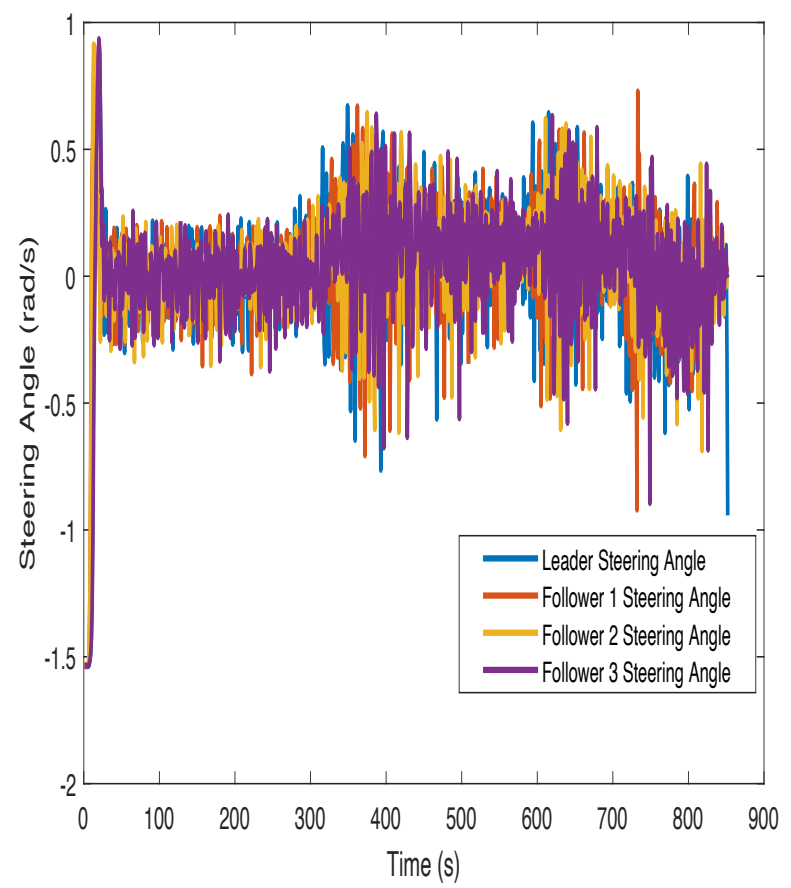

(c)

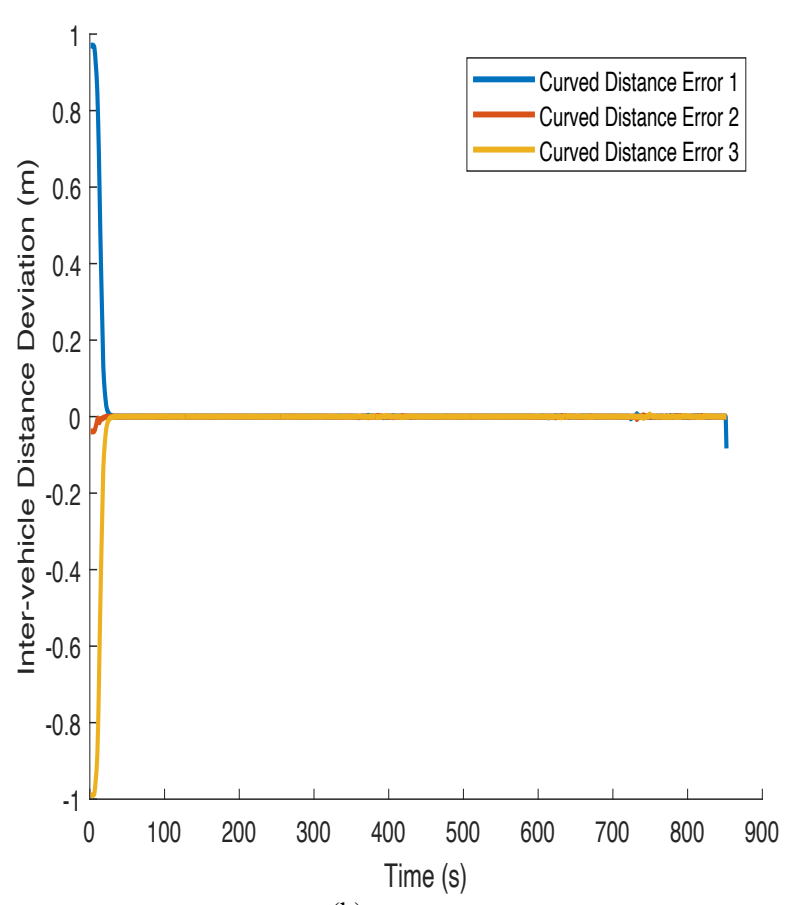

(b)

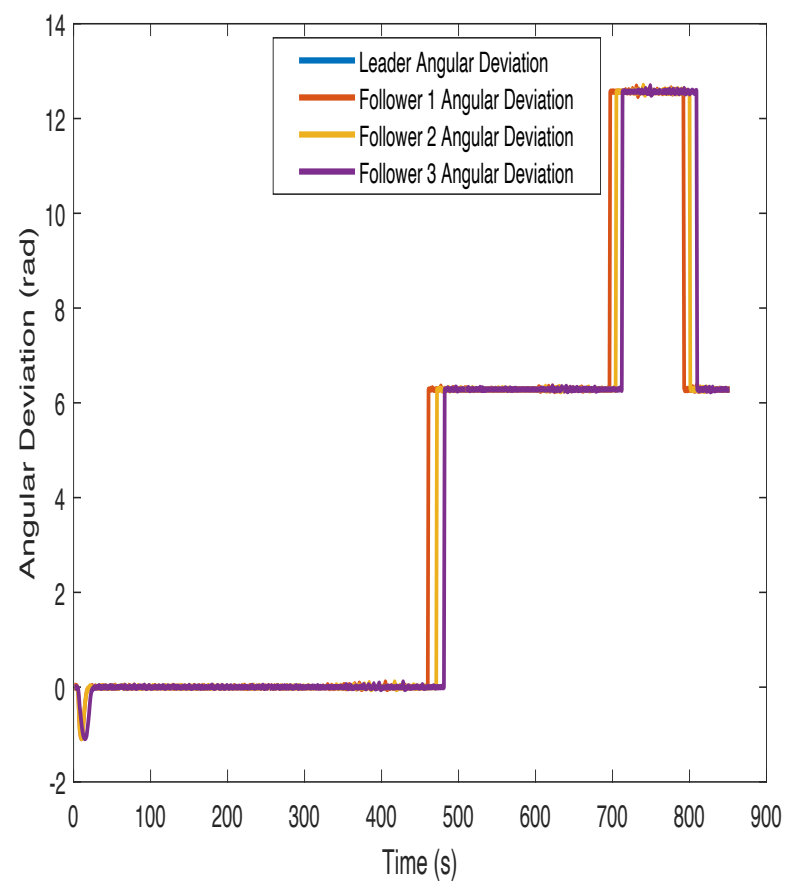

(d)

Figure 6 Decentralized control under PLF: (a)Vehicular velocity; (b)Vehicular distance error; (c)Steering Angle; (d)Angular Deviation. 


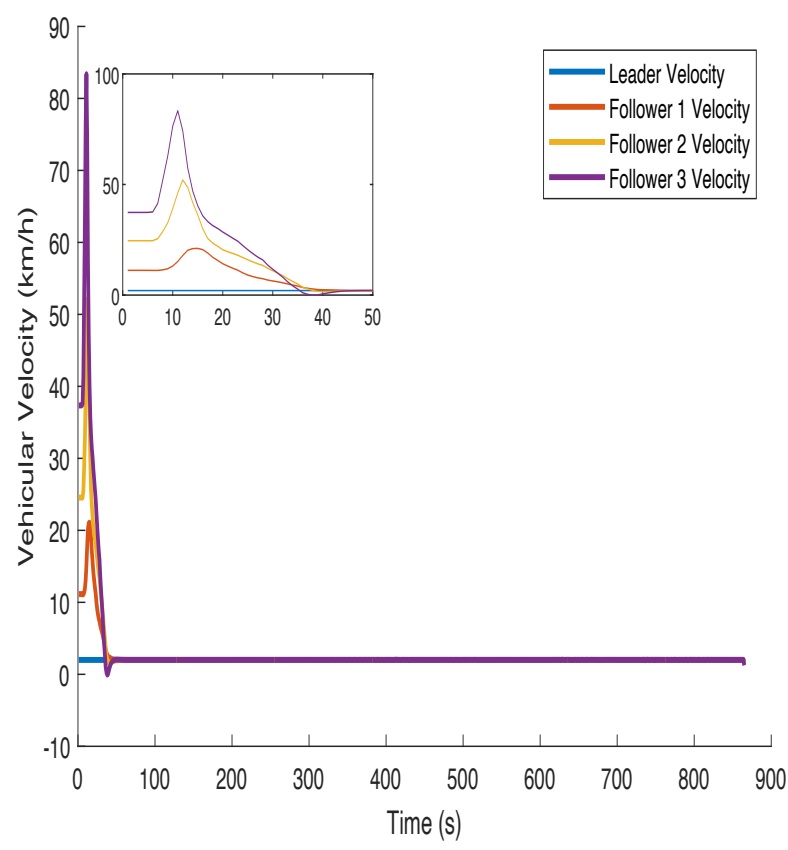

(a)

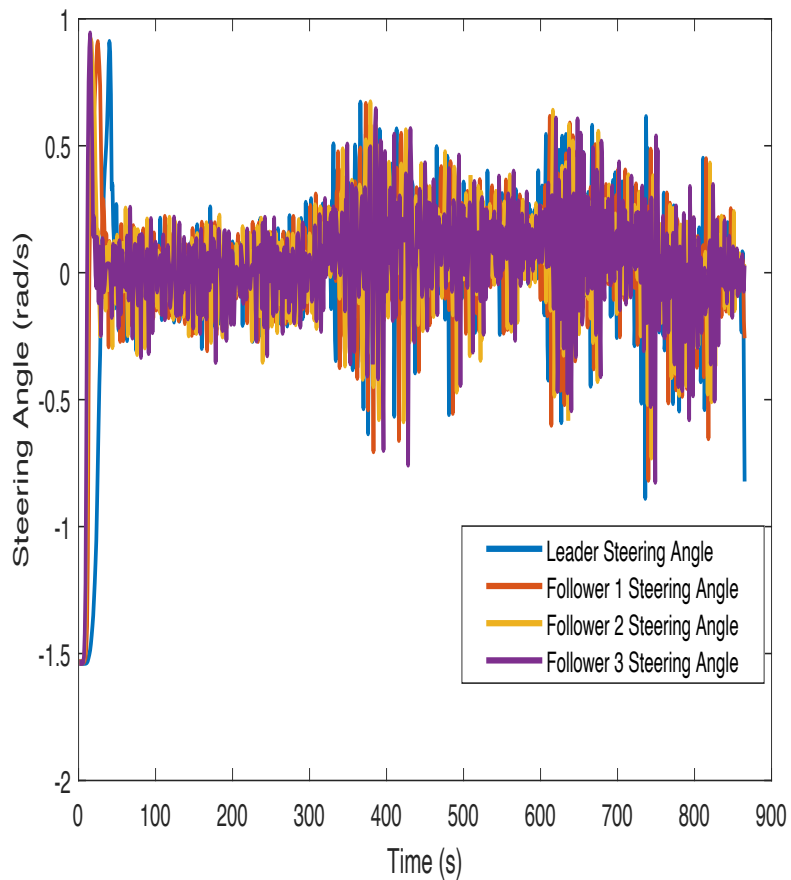

(c)

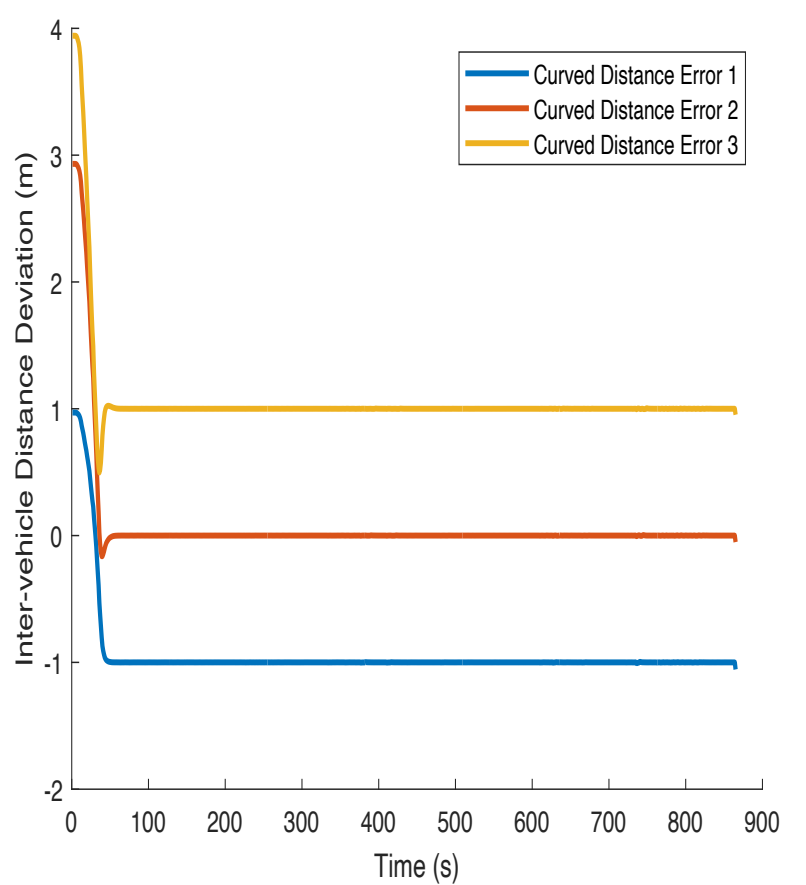

(b)

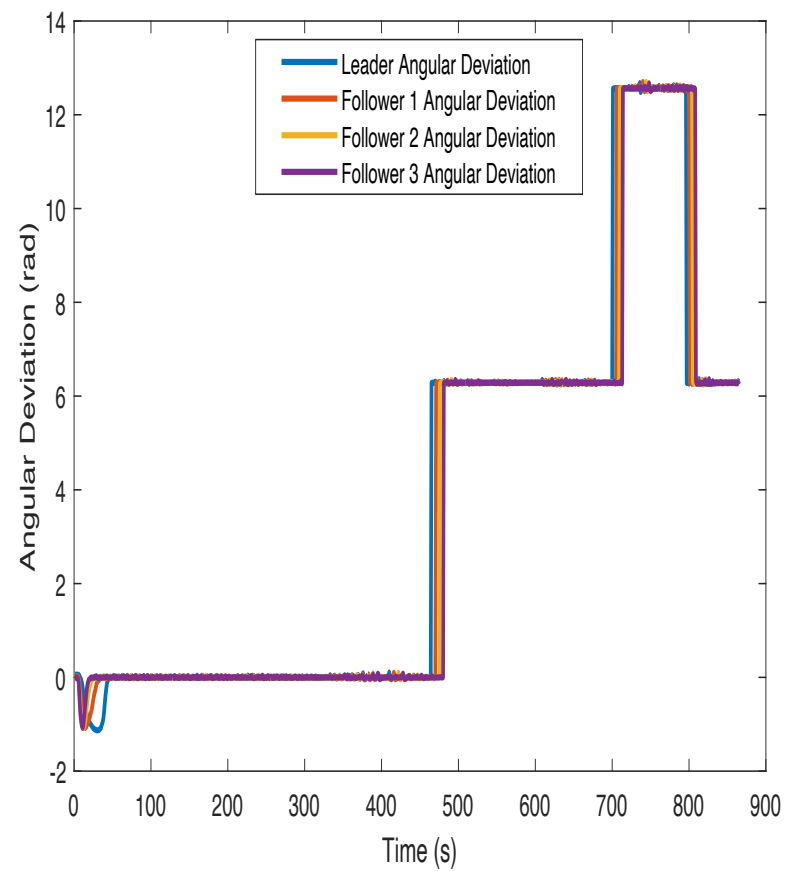

(d)

Figure 7 Centralized control by leader: (a)Vehicular velocity; (b)Vehicular distance error; (c)Steering Angle; (d)Angular Deviation. 


\subsection{Platooning consensus}

Definition 1: An important indicator of vehicular platoon is string stability. A platoon is said to be string stable if the disturbances are not amplified when propagated downstream along the vehicle string [40]. String stability is important to vehicular safe, but it does not represent the consensus of platoon [41]. A platoon is said to be consensus if the follows move like the leader in the same location and the disturbances are not amplified when propagated downstream along the vehicle string. The following are the experimental results of typical platooning control schemes.

After the start of the simulation experiment, the vehicle joins the platoon at a different speed, and the adjustment of the control law $K$ is obtained as shown in Fig. 5(a). Fig. 5 (b) shows that the distance error between all vehicles is zero after the control adjustment. All vehicles are driven to maintain the desired inter-vehicle distance between the vehicles. Fig. 5(c) and 5(d) show that all platoon members follow the preceding vehicle and turn together with it, but the delay time is large. In general, Fig. 5(a) and 5(b) show that the longitudinal motion is consensus, and Fig. 5(c) and 5(d) show that the consensus of lateral motion is poor.

Fig. 6(a) shows that, under the control law $K$, after the platoon member can obtain the leader information, the velocity of the platoon members converges faster than that of Fig. 5(a). Fig. 6(b) shows that the distance error tends to zero from both directions, indicating that the state of motion of the leader has a great influence on the motion state of the tail vehicle. Fig. 6(c) is consensus with the change in the steering angle. Fig. 6(d) not only shows platoon members following the leader vehicle but also quickly responds to the rotation of the leader vehicle. This indicates that the vehicle consensus under the PLF topology is stronger than the vehicle consensus under the PF. Overall, scheme (b) is stronger than scheme (a) in terms of vehicle consensus.

The scheme of Fig. 7 employs centralized control. The result of Fig. 7 is a simulation experiment conducted under reliable communication. Fig. 7(a) is the speed tends to stabilize under the control law $K$. Fig. 7(b) is caused by the failure to obtain information about the motion state of the preceding vehicle. Fig. 7(c) and (d) show that the response speed to the movement of the leader vehicle is better than scheme (a) and worse than scheme (b). This is because although scheme (c) can obtain the moving state of the leader vehicle, it cannot obtain the moving state of the preceding vehicle.

In general, the leader vehicle information and the preceding vehicle information are both important for a consensus platoon. In the process of obtaining the information of the leader vehicle or the preceding vehicle, when the platoon is on the curved road, the inter-vehicle distance is curved. At this time, if the communication transmission power is set according to the constant inter-vehicle distance, battery energy will be wasted. Therefore, we propose communication power transmission with adaptive road curvature.

\subsection{Platooning energy efficiency}

An energy consumption simulation is established to evaluate the energy efficiency of the vehicle on curved road when the vehicle communication equipment sends a platooning control message.

When the platooning vehicle passes the curved road, the relationship between the inter-vehicle distance of communication and the road curvature is shown in Fig. 8.

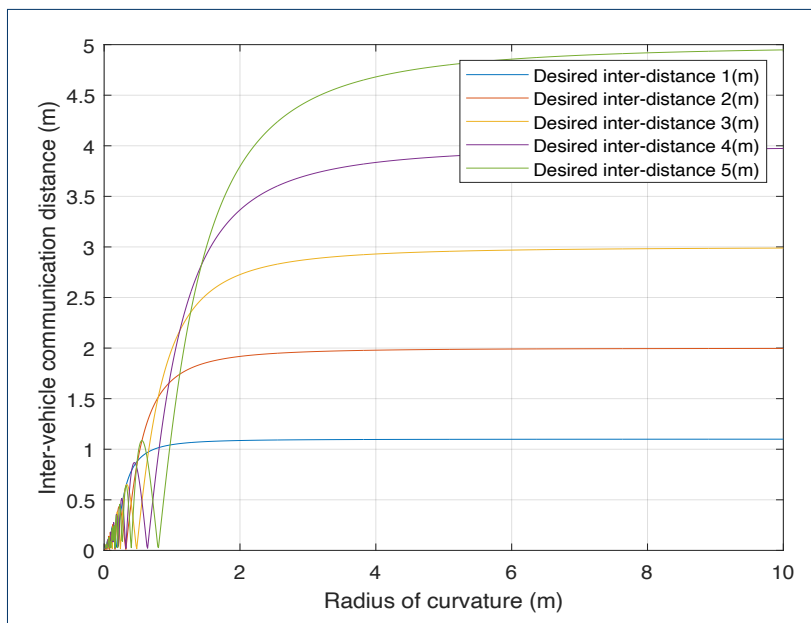

Figure 8 Inter-vehicle communicaiton distance under two-dimensional space.

According to inter-vehicle communication distance, Algorithm 1 is used to reduce communication power consumption in the electric vehicle platoon. The essence of the Algorithm 1 is to adapt radius of road curvature and adjust the transmission power of the preceding vehicle, so as to save more energy on the curved road.

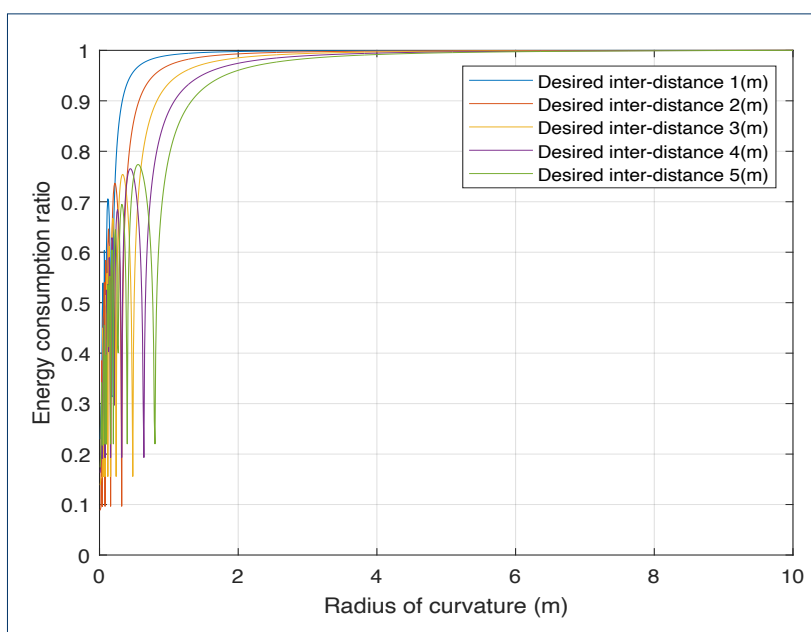

Figure 9 Communication energy consumption under adaptive transmission power. 
On the straight road, the minimum transmission power of preceding vehicles is calculated by path loss equation (12b). When the platooning enters the curved road, the inter-vehicle communication distance of platooning is unequal to the desired inter-vehicle distance. Relative to the minimum transmitted power of vehicles on the straight road, the energy consumption ratio on the curved road is shown in Fig. 9. Simulation results show that the bigger desired inter-vehicle distance, the more power is saved under Algorithm 1. However, the bigger inter-vehicle distance, the worse aerodynamic performance of platoon members. According to a two-car queue measured by University of Southern California scholars in the Dryden Wind Tunnel, when the inter-vehicle distance is $0-5 m$, the actual wind resistance coefficient of the rear vehicle remains basically unchanged and remains at a low level [42]. Combined with the analysis of Fig. 9, the electric vehicle platoon under desired inter-vehicle distance $5 m$ can save $5.3 \%$ of the energy of the communication system.

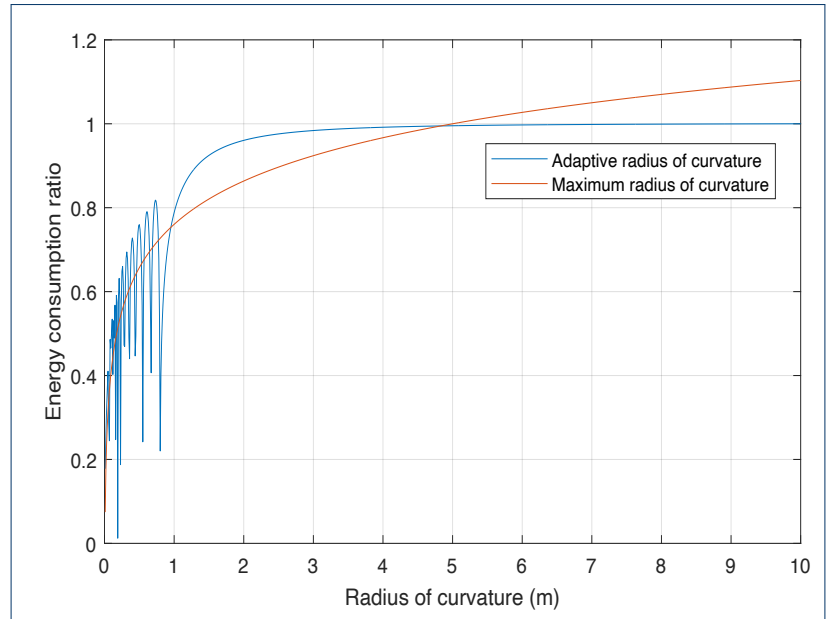

Figure 10 Communication energy consumption under desire inter-vehicle distance $5 \mathrm{~m}$.

Relative to the minimum transmitted power of preceding vehicles on the straight road with the desire inter-vehicle distance of 5m, Fig. 10 shows the energy consumption ratio under the adaptive radius of curvature and a maximum radius of curvature.

When the radius of curvature is less than $5 \mathrm{~m}$, the energy consumption ratio of the former method is larger than that of the latter method, indicating that the latter method can save more energy. When the radius of curvature is greater than $5 \mathrm{~m}$, the energy consumption of the latter method exceeds the straight road energy consumption and continues to increase, indicating that the communication power of the preceding vehicle can not meet the platooning communication needs. From the perspective of the entire curvature change process, the adaptive radius of the curvature method does not increase the transmission power demand as the curvature increases, so that the energy consumption of the communication system is small.

\section{CONCLUSION AND FUTURE WORK}

In the car-like model, this paper analyzes the curve intervehicle distance error and uses a constant control law $K$ to stabilize the platooning. Then, three platooning control schemes are established, and the vehicle dynamics of these three platooning control schemes are simulated. Finally, a communication energy allocation algorithm for adaptive road curvature radius is proposed to optimize the transmission power of preceding vehicles on the curved road. The proposed scheme saves platooning communication system energy of $5.3 \%$ than the platooning without this method on a curved road. Simulation results show that the proposed scheme is better than the other two conventional communication energy schemes.

In future work, we will study the impact of communication on the MES by reducing inter-vehicle communication latency. Reducing the inter-vehicle communication delay can make the vehicle steering more sensitive. Since the power control system can control the vehicle steering with the road curvature accurately and timely, it can reduce unnecessary braking energy consumption and increase platooning cruising range.

\section{Appendix}

Proof of the Equations 2a, $2 \mathrm{~b}$ and $2 \mathrm{c}$

Let $\dot{S}_{\Delta t}^{i}$ is the differential of the distance in time $\Delta t$, and $\dot{D}_{t}^{i}$ is the differential of vehicle $i$ lateral distance at time $t$. According to Fig. 1, we can show that

$\dot{D}_{t}^{i}=v_{i} \cdot \sin \theta_{t}^{i}$

Then

Equation $2 b$ is established.

$$
\begin{aligned}
& \frac{1 / c_{i}}{1 / c_{i}+D_{i}}=\frac{\dot{S}_{\Delta t}^{i}}{v_{i} \cos \left(\gamma_{t}^{i}-\alpha_{t}^{i}\right)} \\
& \Rightarrow \dot{S}_{\Delta t}^{i}=\frac{v_{i} \cos \theta_{t}^{i}}{1+D_{i} c_{i}}
\end{aligned}
$$

Then

Equation 2a is established.

Because $1 / c_{i} \cdot \alpha_{t}^{i}=S_{\Delta t}^{i}$ is definited. Therefore, there must exit

$\alpha_{t}^{i}=S_{\Delta t}^{i} \cdot c_{i}$

$\dot{\alpha}_{t}^{i}=\dot{S}_{\Delta t}^{i} \cdot c_{i}$

Given that $\theta_{t}^{i}=\gamma_{t}^{i}-\alpha_{t}^{i}$ and equation (1c) we can get the equation.

$\Rightarrow \dot{\theta}_{t}^{i}=v_{i}\left(\frac{\tan \left(\gamma_{t}^{i}-\beta_{t}^{i}\right)}{L}-\frac{c_{i} \cdot \cos \theta_{t}^{i}}{1+D_{t}^{i} c_{i}}\right)$

Then

Equation 2c is established.

Proof of the Equation $12 \mathrm{a}$

Let $S+\delta_{\Delta t}^{i}=a \cdot R$

where $S+\delta_{\Delta t}^{i}$ is arc length, $a$ is center angle, and $R$ is radius. Then, 
$\sin (a / 2)=(d / 2) / R$

so, $d=2 R \cdot \sin (a / 2)$

Now,

$a=\left(S+\delta_{\Delta t}^{i}\right) / R$ and $R=1 / c_{i}$

Hence,

$d=\frac{2}{c_{i}} \cdot \sin \left(\frac{\left(S+\delta_{\Delta t}^{i}\right) \cdot c_{i}}{2}\right)$.

\section{Abbreviations}

MES:main energy systems; AES:auxiliary energy systems; MATLAB:matrix laboratory; EVs: electric vehicles; V2V: vehicle to vehicle;

1-D:one-dimensional; PF:predecessor-following; PLF: predecessor- leader following; BD:bidirectional; BDL:bidirectional-leader; TPF:two-predecessors following; TPLF:two-predecessor-leader following; OBU:On board Unit; TPC:transmit power control;

\section{Acknowledgements}

This research was supported in part by The Okawa Foundation for Information and Telecommunications, in part by G-7 Scholarship Foundation, in part by JSPS KAKENHI grant numbers $18 \mathrm{KK} 0279$, $19 \mathrm{H} 04093,20 \mathrm{H} 00592$, and $21 \mathrm{H} 03424$.

\section{Funding}

This work was supported in part by China Scholarship Council.

\section{Availability of data and materials}

All data of simulation and experiment can be obtained by contacting the authors of the paper.

\section{Competing interests}

The authors declare that they have no competing interests.

\section{Authors' contributions}

Both authors in the paper have equal contributions from the idea to the completion of the paper.

\section{Authors' information}

Wei Gao is currently pursuing the Ph.D. degree with the State Key Laboratory of Networking and Switch- ing Technology, Beijing University of Posts and Telecommunications, Beijing, China. His research interests are in the areas of multi-vehicle platoon formation control, and vehicle communication.

Celimuge Wu (celimuge@uec.ac.jp) received his M.E. degree from Beijing Institute of Technology, Beijing, China, in 2006, and PhD degree from the University of Electro-Communications, Tokyo, Japan, in 2010. He was an assistant professor at the Graduate School of Information Systems, the University of Electro-Communications from 2010, and since November 2015 , he has been an associate professor at the same university. He current research interests include vehicular networks, edge Comput- ing, and intelligent transport systems.

\section{Author details}

${ }^{1}$ Beijing University of Posts and Telecommunications Beijing, the State Key Laboratory of Networking and Switching Technology, Beijing, China. ${ }^{2}$ the Department of Computer and Network Engineering, The University of Electro-Communications 1-5-1, Chofugaoka, Tokyo, Japan.

\section{References}

1. Ortuzar, M., Moreno, J., Dixon, J.: Ultracapacitor-based auxiliary energy system for an electric vehicle: Implementation and evaluation. IEEE Transactions on Industrial Electronics 54(4), 2147-2156 (2007). doi:10.1109/TIE.2007.894713

2. Massera Filho, C., Terra, M.H., Wolf, D.F.: Safe optimization of highway traffic with robust model predictive control-based cooperative adaptive cruise control. IEEE Transactions on Intelligent Transportation Systems 18(11), 3193-3203 (2017). doi:10.1109/TITS.2017.2679098

3. 3GPP: 3rd generation partner ship project 22.886; technical specification group services and system aspects; study on enhancement of $3 g p p$ support for $5 g$ v2x services ( $v 16.2 .0$, release 16). (2018)
4. Jia, D., Lu, K., Wang, J., Zhang, X., Shen, X.: A survey on platoon-based vehicular cyber-physical systems. IEEE Communications Surveys Tutorials 18(1), 263-284 (2016). doi:10.1109/COMST.2015.2410831

5. Zheng, Y., Li, S.E., Li, K., Ren, W.: Platooning of connected vehicles with undirected topologies: Robustness analysis and distributed h-infinity controller synthesis. IEEE Transactions on Intelligent Transportation Systems 19(5), 1353-1364 (2018). doi:10.1109/TITS.2017.2726038

6. Li, S.E., Qin, X., Zheng, Y., Wang, J., Li, K., Zhang, H.: Distributed platoon control under topologies with complex eigenvalues: Stability analysis and controller synthesis. IEEE Transactions on Control Systems Technology 27(1), 206-220 (2019). doi:10.1109/TCST.2017.2768041

7. Seiler, P., Raja Sengupta: An h/sub /spl infin// approach to networked control. IEEE Transactions on Automatic Control 50(3), 356-364 (2005). doi:10.1109/TAC.2005.844177

8. Thibault, L., De Nunzio, G., Sciarretta, A.: A unified approach for electric vehicles range maximization via eco-routing, eco-driving, and energy consumption prediction. IEEE Transactions on Intelligent Vehicles 3(4), 463-475 (2018). doi:10.1109/TIV.2018.2873922

9. Zhang, S., Luo, Y., Wang, J., Wang, X., Li, K.: Predictive energy management strategy for fully electric vehicles based on preceding vehicle movement. IEEE Transactions on Intelligent Transportation Systems 18(11), 3049-3060 (2017). doi:10.1109/TITS.2017.2672542

10. Xu, W., Chen, H., Wang, J., Zhao, H.: Velocity optimization for braking energy management of in-wheel motor electric vehicles. IEEE Access 7, 66410-66422 (2019). doi:10.1109/ACCESS.2019.2915102

11. Kachroudi, S., Grossard, M., Abroug, N.: Predictive driving guidance of full electric vehicles using particle swarm optimization. IEEE Transactions on Vehicular Technology 61(9), 3909-3919 (2012). doi:10.1109/TVT.2012.2212735

12. Jin, C., Sheng, X., Ghosh, P.: Optimized electric vehicle charging with intermittent renewable energy sources. IEEE Journal of Selected Topics in Signal Processing 8(6), 1063-1072 (2014). doi:10.1109/JSTSP.2014.2336624

13. Khalifa, A., Kermorgant, O., Dominguez, S., Martinet, P.: Platooning of car-like vehicles in urban environments: An observer-based approach considering actuator dynamics and time delays. IEEE Transactions on Intelligent Transportation Systems, 1-13 (2020)

14. Godbole, D.N., Lygeros, J.: Longitudinal control of the lead car of a platoon. IEEE Transactions on Vehicular Technology 43(4), 1125-1135 (1994)

15. Wei, S., Zou, Y., Zhang, X., Zhang, T., Li, X.: An integrated longitudinal and lateral vehicle following control system with radar and vehicle-to-vehicle communication. IEEE Transactions on Vehicular Technology 68(2), 1116-1127 (2019)

16. Bayuwindra, A., Ploeg, J., Lefeber, E., Nijmeijer, H.: Combined longitudinal and lateral control of car-like vehicle platooning with extended look-ahead. IEEE Transactions on Control Systems Technology 28(3), 790-803 (2020)

17. Ghasemi, A., Kazemi, R., Azadi, S.: Stable decentralized control of a platoon of vehicles with heterogeneous information feedback. IEEE Transactions on Vehicular Technology 62(9), 4299-4308 (2013)

18. Yadlapalli, S.K., Darbha, S., Rajagopal, K.R.: Information flow and its relation to stability of the motion of vehicles in a rigid formation. IEEE Transactions on Automatic Control 51(8), 1315-1319 (2006)

19. Sadraddini, S., Sivaranjani, S., Gupta, V., Belta, C.: Provably safe cruise control of vehicular platoons. IEEE Control Systems Letters 1(2), 262-267 (2017)

20. Chehardoli, H., Ghasemi, A.: Adaptive centralized/decentralized control and identification of 1-d heterogeneous vehicular platoons based on constant time headway policy. IEEE Transactions on Intelligent Transportation Systems 19(10), 3376-3386 (2018). doi:10.1109/TITS.2017.2781152

21. Wu, H.-x., Cheng, S.-k., Cui, S.-m.: Communication of vehicle management unit in the electric vehicle. IEEE Transactions on Magnetics 41(1), 514-517 (2005). doi:10.1109/TMAG.2004.839273

22. Zander, J.: Performance of optimum transmitter power control in cellular radio systems. IEEE Transactions on Vehicular Technology 41(1), 57-62 (1992). doi:10.1109/25.120145 
23. Grandhi, S.A., Vijayan, R., Goodman, D.J., Zander, J.: Centralized power control in cellular radio systems. IEEE Transactions on Vehicular Technology 42(4), 466-468 (1993). doi:10.1109/25.260766

24. Rosberg, Z., Zander, J.: Toward a framework for power control in cellular systems. Wireless Networks 4(4), 215-222 (1998)

25. Sommer, C.: Veins. The open source vehicular network simulation framework (2006-2020)

26. Segata, M.: Plexe. The Platooning Extension for Veins (2014-2018)

27. Li, S.E., Zheng, Y., Li, K., Wang, J.: An overview of vehicular platoon control under the four-component framework. In: 2015 IEEE Intelligent Vehicles Symposium (IV), pp. 286-291 (2015). doi:10.1109/IVS.2015.7225700

28. Hao, H., Barooah, P., Mehta, P.G.: Stability margin scaling laws for distributed formation control as a function of network structure. IEEE Transactions on Automatic Control 56(4), 923-929 (2011). doi:10.1109/TAC.2010.2103416

29. Peng, J.Z.: Range policy of adaptive cruise control vehicles for improved flow stability and string stability. IEEE Transactions on Intelligent Transportation Systems 6(2), 229-237 (2005). doi:10.1109/TITS.2005.848359

30. Liang, C., Peng, H.: Optimal adaptive cruise control with guaranteed string stability. VEHICLE SYSTEM DYNAMICS 32(4-5), 313-330 (1999). doi:10.1076/vesd.32.4.313.208

31. Zheng, Y., Eben Li, S., Wang, J., Cao, D., Li, K.: Stability and scalability of homogeneous vehicular platoon: Study on the influence of information flow topologies. IEEE Transactions on Intelligent Transportation Systems 17(1), 14-26 (2016). doi:10.1109/TITS.2015.2402153

32. Li, S.E., Zheng, Y., Li, K., Wu, Y., Hedrick, J.K., Gao, F., Zhang, H.: Dynamical modeling and distributed control of connected and automated vehicles: Challenges and opportunities. IEEE Intelligent Transportation Systems Magazine 9(3), 46-58 (2017). doi:10.1109/MITS.2017.2709781

33. Rajamani, R., Han-Shue Tan, Boon Kait Law, Wei-Bin Zhang: Demonstration of integrated longitudinal and lateral control for the operation of automated vehicles in platoons. IEEE Transactions on Control Systems Technology 8(4), 695-708 (2000). doi:10.1109/87.852914

34. Ali, A.: Modeling and control of a platoon of urban autonomous vehicles. PhD thesis, :Research Institute in Communications and Cybernetics of Nantes (IRCCyN) (2015)

35. M. Segata, H.M.C. T. R. Li: Safe and efficient communication protocols for platoon control. PhD Thesis (Dissertation) University of Innsbruck (2016)

36. Segata, M., Joerer, S., Bloessl, B., Sommer, C., Dressler, F., Cigno, R.L.: Plexe: A platooning extension for veins. In: 2014 IEEE Vehicular Networking Conference (VNC), pp. 53-60 (2014). doi:10.1109/VNC.2014.7013309

37. Wang, S..D.W..v.A.B..S.B. M ; Hoogendoorn: Delay-compensating strategy to enhance string stability of adaptive cruise controlled vehicles. TRANSPORTMETRICA B-TRANSPORT DYNAMICS 6(3), 211-229 (2018). doi:10.1080/21680566.2016.1266973

38. Wei, L., Hu, R.Q., Qian, Y., Wu, G.: Energy efficiency and spectrum efficiency of multihop device-to-device communications underlaying cellular networks. IEEE Transactions on Vehicular Technology 65(1), 367-380 (2016). doi:10.1109/TVT.2015.2389823

39. 3GPP: 3rd generation partnership project 38.901 ; technical specification group radio access network; study on channel model for frequencies from 0.5 to $100 \mathrm{ghz}$ (release 16). (2019)

40. Swaroop, D., Hedrick, J.K.: String stability of interconnected systems. In: Proceedings of 1995 American Control Conference - ACC'95, vol. 3, pp. 1806-18103 (1995). doi:10.1109/ACC.1995.531196

41. Santini, S., Salvi, A., Valente, A.S., PescapšŠ, A., Segata, M., Lo Cigno, R.: A consensus-based approach for platooning with intervehicular communications and its validation in realistic scenarios. IEEE Transactions on Vehicular Technology 66(3), 1985-1999 (2017). doi:10.1109/TVT.2016.2585018

42. Hammache, M., Michaelian, M., Browand, F.: Aerodynamic forces on truck models including two trucks in tandem. SAE Paper 1(0530) (2002) 